\title{
Bio-Magnetoplasmonics, emerging biomedical technologies and beyond
}

Volume 3 Issue 3 - 2016

\section{Mini Review}

Abbreviations: BMP, Bio-Magnetoplasmonics; MP, Magnetoplasmonics; SPs, Surface Plasmons; EM, Electromagnetic; SPR, Surface Plasmon Resonance; SPP, Surface Plasmon Polaritons; LSPs, Localized Surface Plasmons; MO, Magneto-Optical; FM, Ferromagnetic

\section{Introduction}

Bio-magnetoplasmonics (BMP), is a relatively new field of science that employs magnetoplasmonics (MP), in biology which has a great potential application in biomedicine and biomedical technologies such as ultrafast and ultra-sensitive biosensing and bio-detection, bioimaging, bio-therapy, drug-delivery, nano-imaging, etc., ${ }^{1-4}$ It merges the physics of bio-nanomagnetics where biological samples such as cells and DNA are made to interact with magnetic moments, M, of a magnetic material or with applied magnetic field, $\mathrm{H}$ and bio-nanooptics where biological samples are made to interact with optical radiation in visible, Infrared and telecommunication wavelength ranges. ${ }^{5-9}$ In a similar manner, it merges bio-nanoplasmonics where biological samples are made to interact with surface plasmonic wave fields, also referred to as evanescent radiation fields.

The circle with big arrows (clockwise) shows a process involved in creating new MP material through innovative design, modeling, simulation and verification of these designs through nanofabrication, optical and magnetic characterization and data fitting. The outer circle (clockwise) shows the various functionalities of MPs - including biosensing, bio-imaging, Immunoassays monitoring of environmental problems, thermal therapy, space exploration, biophysics and photovoltaics. Schematic of essential characteristics of nanoparticles used in magnetoplasmonic is shown in the top right and that of multilayered nanostructure in the bottom right.

Surface plasmons (SPs) are oscillating charge density waves and are created on the surface of the materials when excited by electromagnetic (EM) radiation. Surface plasmon resonance (SPR) occurs when the wave-vector of the incident optical radiation matches that of the wave vector of the surface plasmons. ${ }^{10-15}$ Much in the same way as the SPR, in magnetoplasmonics, in the presence of magnetic materials or external magnetic fields

a. Plasmonics is used to enhance the magnetic response (e.g. Kerr effect) and

b. Magnetism is used as a way to make plasmonics tunable with a modest external magnetic field or direction sensitive. When the plasmon waves are coupled with the incident optical radiation, the effect arises due to the spin-orbit interaction and Lorenz force drift as $\mathrm{H}$ fields are introduced.

Depending on the device geometry and sizes, there are two types of SPs. One is the localized surface plasmons (LSPs), which are nonpropagating excitation of the plasmons coupled to the EM fields and

\author{
Conrad Rizal,' Boris Niraula, ${ }^{2}$ Howard Ho \\ Wai Lee, ${ }^{3,4}$ \\ 'University of California, USA \\ ${ }^{2}$ University of British Columbia, Canada \\ ${ }^{3}$ Baylor University, USA \\ 4Texas A\&M University, USA
}

Correspondence: Conrad Rizal, University of California, San Diego, La Jolla, CA, 92093, USA, Tel (858) 433-6788 Email c.s.rizal@gmail.com

Received: January 22, 2016 | Published: April 0I, 2016

confined within the nanostructures such as metallic nanoparticles, nanorods, nanodisks, nanowires, etc., whose dimensions are smaller than the wavelength of the incident optical radiation. ${ }^{5,11,15-19}$ The other type is the surface plasmon polaritons (SPP), which arises from the coupling of the electrons at the interface between the materials with negative and positive permittivity (which is the case for metal and dielectric layers) and incident EM waves produces oscillations of electron plasma on the metallic surface that in turn generates confined two-dimensional transverse EM waves at the interface. ${ }^{2,20-25}$ These waves decay exponentially in the direction perpendicular to the interface and practically vanish at both sides of the interface. Unlike the LSPs, the SPP arises from the layered nano structured configurations where the lateral size of the nanostructure is greater than the wavelength of the optical radiation such as, multilayers of dielectric/ferromagnetic, dielectric/oxides and dielectric/plasmonic metals.

Commonly available and most popular materials used in plasmonic studies for SPR phenomena are non-magnetic (NM) noble metals such as, $\mathrm{Au}, \mathrm{Cu}$ and $\mathrm{Ag}$. However, being purely NM, the optical properties are determined in an extended spectral range by the conduction electron and as determined by the Drude's theory of conduction of electron in metals, they exhibit small magneto-optical (MO) effects. The 3-d transition ferromagnetic (FM) metals such as $\mathrm{Co}, \mathrm{Fe}$ and $\mathrm{Ni}$ and ferri-magnetic metals and their alloys and multilayers are excellent candidates for the study of MO surface plasmon resonance (MO-SPR); primarily because they offer excellent $\mathrm{MO}$ and optical properties such as enhanced permittivity and electromagnetic coupling and these can be controlled using both optical radiation and $\mathrm{H}$ fields. It is a well established fact that $\mathrm{FM}$ of $\mathrm{Fe}, \mathrm{Co}$ and $\mathrm{Ni}$ offer large MO effects (e.g., MO effects in Co is 1000 times larger than in NM metals such as Au). The propagation length of SPP calculated from Maxwell's equation for NM metals extends in the ranges of 100$\mathrm{s}$ of micrometers compared to just below 10 micrometers for the FM metals such as Co. It implies that FM metals alone cannot be used as the only metallic components of the MP system, but they can be embedded with the NM metals or oxides in the form of Core-shell 
or multilayered structure to increase MO effects and propagation length of the SPP. A summary of design of new MP structure and the various processes involved with these as well as about the potential applications of MPs is schematically demonstrated in Figure 1.

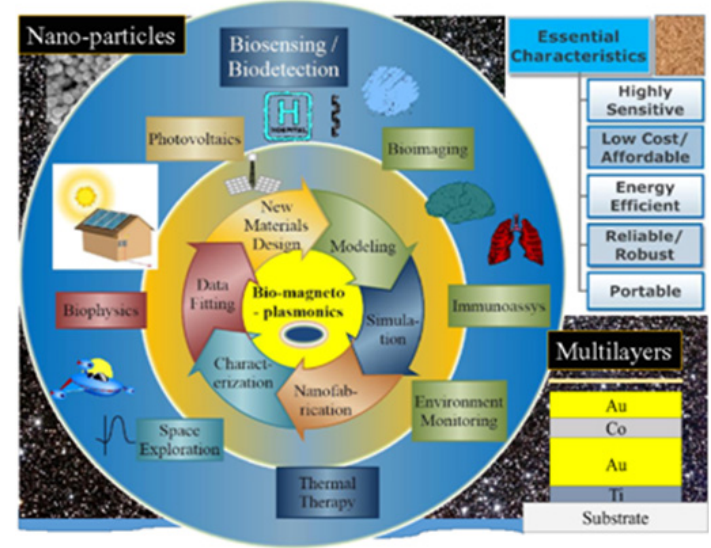

Figure I Schematic representation of various processes that are currently being studied within the field of magneto-plasmonics (MP) (inner circle).

SPR-based sensing using NM metals have already found commercial applications. However, detecting low concentration of biomolecules using SPR sensors is still a challenge as ultra-sensitive sensors are required for the early detection and treatment of diseases such as AIDS, tropical fevers, cancers, etc. and the signal to noise ratio of SPR sensors is limited. ${ }^{21,26,27}$ If modified by suitable magnetic materials, it is one of the most important areas where MO-SPR sensors would shine as ultra-fast, miniature and ultra-sensitive bio-sensor for early detection of chronic diseases. Since magnetic switching in FM multilayer can be achieved through optical radiation. ${ }^{28}$ and this interplay between the optical radiation and magnetic moments in the FM multilayer enables one to modify MO properties leading to high sensitivity, especially in reference to changes in refractive indices and permeability of the biological samples and the metal layers involved. For a detail review on these magnetic multilayered-based magnetoplasmonic systems, interested readers are suggested to read review articles and exciting work on MP carried out by us. ${ }^{29-31}$ as well as by Armelles and his research groups. ${ }^{2}$

The study of layered metal/dielectric-based plasmonics is a relatively new science and promises with many potential new applications in biomedicine. We recently explored new ways to enhance MO and optical modulation respectively using new ferromagnetic and dielectric layers. ${ }^{29,32}$ and theoretically demonstrated that only under an optimum design conditions, an incident optical radiation can couple to selected surface plasmon polaritons modes through the synergetic action between optical radiation and magneto-optically induced fields. This required using $\mathrm{H}$ fields to change the direction of magnetic moment in the sample and optical radiation at various incident angle, as external stimuli. ${ }^{28}$ It also involved miniaturization and optimization of the device structure. Combination of all these efforts lead to an increase in sensitivity of the biosensor to two order of magnitude as opposed to those conventional SPR based sensors.

Figure 2 (i) shows $\mathrm{Au} /$ dielectric-plasmonic nanostructures we have designed, optimized and fabricated. The SPR shift achievable by our structure is shown in Figure 2 (ii), where the minimum reflection peak shifts towards a higher $\theta$ for a protein sample. The angular shift, corresponding to the changes in $\varepsilon$, defines the sensitivity of the SPR configuration. Our modeling shows that the sensitivity of the SPR is not sufficient to detect a sample that has a very low protein concentration.
Figure 2 (iii) shows the schematic of the new MOSPR structure that we recently designed, optimized and fabricated and Figure (iv) is the MOSPR effect shown by the multilayer. Instead of investigating the linear angular shifts in the SPR peaks, here we record the changes in reflectivity intensity corresponding to the changes in the permittivity of the sample when an $\mathrm{H}$ field is introduced. The sharp changes in the MOSPR curves result in the giant magneto-reflection (GMR) effect, defined as: $[\mathrm{Rp}(\mathrm{H}=+\mathrm{H})-\mathrm{Rp}(\mathrm{H}=-\mathrm{H})] / \mathrm{Rp}(\mathrm{H}=0)$, where $\mathrm{Rp}(\mathrm{H}=+$ $H), R p(H=-H)$ and $R p(H=0)$ denote reflections at positive, negative and zero applied $\mathrm{H}$ fields. Due to the tunability of light and magnetic fields, this sensor configuration can detect as small as 100 parts per billion changes in material optical properties that is 2 orders more magnitude changes in sensitivity of conventional SPR configuration. That means that, if suitably designed, fabricated and optimized, the MOSPR configuration is highly suitable for detecting samples that have extremely low protein concentration. The increased sensitivity of the MOSPR sensor is attributed to the amplified MO signal, induced by the excitation of the SPPs at the metal and dielectric interface, in response to the applied transverse $\mathrm{H}$ fields (the direction, as shown in the inset of Figure (iv)) and transverse-magnetic polarized optical radiation. Also, Figure (v-vii) show the light enhancement in nonmagnetic $\mathrm{Ag} / \mathrm{Si}$ multilayers that can be extended to ferromagnetic MP nanostructures for potential biomedical applications.

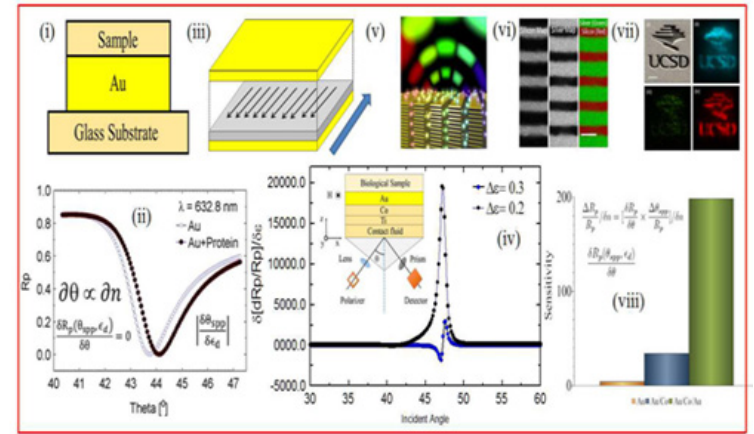

Figure 2 SPR and MOSPR effects:

i. Conventional SPR structure,

ii. Reflectivity vs incident angle,

iii. MOSPR structure,

iv. Sensitivity $V_{s}$ incident optical angle of radiation. The inset in

v. shows the stimuli setup - It consists of basic MOSPR configuration for biosensing: A indexing matching fluid is placed in between the triangular prism and Ti buffer layer, containing $45 \mathrm{~nm}$ Au/Co multilayer mounted on a prism. Incident optical radiation is fed through the prism and buffer layer, and is reflected off the multilayer that passes back through the prism to a photodetector where it is collected. The changes in the reflectivity intensity versus incident angle of radiation is directly linked to the refractive index of the bio-sample attached to the multilayer surface.

vi. Optical emission enhancement in $\mathrm{Ag} / \mathrm{Si}$ multilayers.

vii. Sensitivity comparison between the SPR and MOSPR configurations. ${ }^{31,32}$

We find that these new nano-structures show distinctly new MP characteristics due to the difference in band diagrams, conductivity profile, carrier concentration and carrier mobility, Ohmic loss and magnetic orientation, to name a few. For a theory on MO layered structures, readers are suggested to read the paper by Schburt et al. ${ }^{33}$

In this mini review, the aim is to provide an overview of many recent pioneering developments in the field of nanoparticles and 
layered structure based surface plasmonics and magnetoplasmonics. The application of metal based SPR sensors and arrays of nano holes are showing ever growing importance of this field, see, for example, the recent works by Ertorer..$^{34}$ and Menezes \& Brolo. ${ }^{11,35}$ The materials and devices are equally important to study the interaction of bio-molecules with magnetoplasmonics and it is so when suitably fabricated. For example, by modifying SPR structure with the magnetic material and by varying the device structure and miniaturization, a new generation of MP material and capabilities can be created, extending the use of plasmon materials in new areas such as - optical radiation-receptor interaction, DNA hybridization, antibody characterization, epitopes mapping, label-free immuno-assay and biomarkers for diseases identification.

Photovoltaics is another area where both the light emission enhancement and extinction features have been already demonstrated using non-magnetic multilayers and metamaterial in grating configurations and this can be extended to FM multi layers based periodic arrays of nano holes. ${ }^{34,35}$

MPs create new opportunities to explore new ways to tune Surface Enhanced Raman Scattering (SERS) using magnetic core-shell nanostructures for the following: Single molecule detection, catalyzed analytical assay, in-situ ambient analysis, protein and nucleic acid, fluorescence properties, imaging, analytical genomic and proteomic studies - drug screening, immunological. A pioneering work on SERS is discussed by Brolo and his research groups. ${ }^{36}$

The application area of new MP structures is enormous. For example, new nano-magnetic structures in various geometries and configurations can be applied to MP nanoscopy (near-field scanning optical microscopy, NSOM), biomedical tests, national security, environmental monitoring, thermal phototherapy of tumours to kill cancer cells (currently on clinical trials), hydrogen sensing (using magnetic core-shell nanoparticles, for example).The use of nanomagnetic materials and ultra-fast magnetic control allows us to maximise the sensitivity, increase detection level and optical energy trapping and heating, to name a few.

Maksymov. ${ }^{3}$ and Vavasari et al. ${ }^{4}$ have recently reviewed work on MO phenomena in MP antenna such as nano-disks, nanorods, etc., that emit, receive and, control optical radiation at the sub-wavelength scale and their sizes are much smaller than the wavelength of the incident optical radiation. The fabrication method employed to grow these nanoantena is compatible with the already established complementary metal-oxide semiconductor (CMOS) manufacturing process, meaning that these can be fabricated with the industry standard CMOS fabrication process with ease. The beauty of these MP nano-antennas are that they have significant potential to be used as novel plasmon rulers and biosensors. Interested readers in review of the origin of the plasmon-enhanced $\mathrm{MO}$ effect are referred to research paper by Prashant $\&$ Cohen et al. ${ }^{18}$

Another MP-localised SPRs of significant interest are MO crystals - artificial structures composed of dielectric and magnetic materials with different refractive indices-that significantly affect the propagation of optical radiation. Innoe et al. ${ }^{1}$ have provided detailed theoretical reviews as well as some experimental works on MO nanocrystals. The magnetic element provides additional degree of freedom to control diffraction patterns, direction and polarization states of optical radiation, in addition to a large enhancement of Faraday rotation in materials consisted of garnet films and dielectric materials. The review also elucidates Bragg grating magnetic $2 \mathrm{D}$ and 3D photonic crystal based theory.
Maccaferri et al.u ${ }^{37}$ have discussed details about recent work on systems allowing label-free molecular detection using MPs that have found some applications in biosensing and are expected to have enormous impact on BMP and biomedicine and deserve intense investigation in this area. We expect that especially, MPbased materials using layered structures will open further many new unforeseen phenomena and functionalities and applications to a variety of emerging technologies, especially in biomedicine and related biomedical fields. Other new devices with improved performance that could come out of MPs are light polarization rotators and non-reciprocal optical isolators that are essential building blocks in bio-nano-photonics technology. A review by Kavanagh \& Gordon. ${ }^{38}$ Chin..$^{39}$ and Tomnov. ${ }^{40}$ highlights details on the control of the non-reciprocal light propagation displayed by metallic and magnetoplasmonic nano-antennas and they could offer a promising route to bring these devices to the nanoscale.

The MP Layered-based devices have already generated significant interest and have demonstrated phenomena such as the giant magnetoreflectence (GMRE) effect, (similar to the giant magnetoresistance (GMR) effect shown by alloys and multi layers.$^{30}$ it is a large change in reflectance, both in amplitude and phase, due to applied magnetic fields) as confirmed by our theoretical modelling and experimental work, shown in Figure 2 (for recent GMR effect and high-saturation magnetization based biosensors, interested readers are referred to papers elsewhere. ${ }^{41-52}$ ). The studies of MP devices and structures using FM, NM and the combination of these two, as well as using insulators and their hybrids with FM material in the form of nano structured multilayers of different configurations and geometry are believed to prompt the efforts in developing magneto-optic surface plasmon (MOSPR) amplification by stimulated emission of radiation and construction of meta-material structure at the nanoscale. Recently, Al, highly doped oxides and graphene are also being used as plasmonic materials as alternatives to $\mathrm{Ag}$ and $\mathrm{Au}$ and these can be incorporated with FM metals to create new MP materials. ${ }^{53-55}$ Since both the light emission enhancement and extinction features have been already demonstrated by non-magnetic multilayers and metamaterial in grating configurations, it would be interesting to see how these effects would change when conducting oxides, e.g. ITO and IZO, are introduced into the new plasmonic nanostructure..$^{14}$ the MPs possess ultra-fast switching behaviour due to controllable spin as well as being extra sensitive to applied $\mathrm{H}$ fields.

\section{Conclusion}

In summary, we introduced recently reviewed series of papers on surface and magneto plasmon structures, including our own. We believe that the review provided a fair and compressive revision of accomplishments in these research fields and about the scope and potential application of magnetoplasmonics. It is a well acknowledged fact that the future of magneto-plasmonic based nanostructures is extremely bright. The exceptional properties displayed by magnetoplasmonic based nanostructures, such as strong enhancement of electromagnetic fields, high sensitivity, large signal to noise ratio, possibility of obtaining high photo-thermal conversion efficiencies and rich spectral responses at applied $\mathrm{H}$ fields, make them unique, outstanding and sought for material for various applications. Their potential application may range from diagnostics, clinical therapy, bioimaging, biophysics, environmental monitoring, ultra-sensitive and ultra-fast molecular sensing for early disease detection, chemical and biological sensing, magneto-plasmon-enabled photo-thermal therapy, magneto-plasmon-assisted laser welding, plasmon-assisted photoacoustic imaging and magneto-plasmon-enhanced spectroscopies, 
such as the SERS for magneto-plasmonic structures and the field is expected to extend to the energy production and as well as in space exploration.

Additional areas where MP-based devices and structures shine are magneto-plasmon enhanced photo-detectors, isolators, solar energy harvesting and conversion and coupling of magnetoplasmons to chemical reactions for achieving high activity and selectivity for energy-saving, femto second switching and sensing and tuning of magneto-optical properties at the femtosecond speed. These areas are highly promising to reach their full potential in new magnetoplasmonic-based technologies - including development of bio-nanomagnetic and magnetoplasmonic bioengineering, highperformance magnetronic devices and green energy, and biology and biomedicine, among many others.

\section{Acknowledgments}

C. Rizal is grateful for excellent feedback and comments received from Eric Fullerton, Center for Magnetic Memory Research, UC San Diego, in the USA.

\section{References}

1. Inoue M, Khanikaev A, Baryshev A "Nano-magnetophotonics". Nanoscale Magnetic Materials and Applications, pp. 2009;627-659.

2. Armelles G, Cebollada A, García-Martín A Magnetoplasmonics: Combining Magnetic and Plasmonic Functionalities. Advanced Optical Materials. 2013;1(1):10-35.

3. Maksymov IS Magneto-plasmonic nanoantennas: Basics and applications (review). ArXiv Preprint arXiv. 2016

4. Lodewijks K, Maccaferri N, Pakizeh T et al. Magnetoplasmonic design rules for active magneto-optics. Nano Letters 2014;14(12):7207-7214.

5. Manera MG, Ferreiro-Vila E, Garcia-Martin JM et al. Enhanced antibody recognition with a magneto-optic surface plasmon resonance (MO-SPR) sensor. Biosensors and Bioelectronics 58: 114-120.

6. Firby C, Elezzabi A Design of integrated YIG-based isolators and highspeed modulators. Presented at SPIE OPTO. 2016

7. Han Z, Elezzabi A, Van V Experimental realization of subwavelength plasmonic slot waveguides on a silicon platform. Opt Lett. 2010;35(4):502-504.

8. Baron C, Elezzabi A Active plasmonic devices via electron spin. Optics Express 17(9): 117-7129.

9. Chau K, Johnson M, Elezzabi A (Electron-spin-dependent terahertz light transport in spintronic-plasmonic media. Phys Rev Lett. 2007; 98(13):133901.

10. Blow N Proteins and proteomics: Life on the surface. Nature Methods. 2009;6(5):389-393.

11. Brolo AG Plasmonics for future biosensors. Nat Photon 6:709-713.

12. Schasfoort RB, Tudos AJ Handbook of Surface Plasmon Resonance. 2008

13. Kihm KD Near-Field Characterization of micro/nano-Scaled Fluid Flows. 2011

14. Lee HW, Papadakis G, Burgos SP et al. Nanoscale conducting oxide Plas MOStor. Nano Letters. 2014;14(11):6463-6468.

15. Anker JN, Hall WP, Lyandres $\mathrm{O}$ et al. Biosensing with plasmonic nanosensors. Nature Materials. 2008;7(6):442-453.

16. Stockman MI Nanoplasmonics: The physics behind the applications. Phys Today. 2011;64(2):39-44.
17. Devkota J, Wang C, Ruiz A et al. Detection of low-concentration super paramagnetic nanoparticles using an integrated radio frequency magnetic biosensor. Journal of Applied Physics. 2013;113(10):104701.

18. Jain PK, Xiao Y, Walsworth R, Cohen AE Surface plasmon resonance enhanced magneto-optics (SuPREMO): Faraday rotation enhancement in gold-coated iron oxide nanocrystals. Nano Letters. 2009;9(4):1644-1650.

19. Xu H, Hajisalem G, Steeves GM et al. Nanorod surface plasmon enhancement of laser-induced ultrafast demagnetization. Scientific Report. 2015;5:15933.

20. Temnov $\mathrm{V}$ The marriage of plasmonics, magnetism, acoustics and ultrafast optics. ArXiv Preprint arXiv pp. 2012;728-736.

21. Sepúlveda B, Calle A, Lechuga LM et al. Highly sensitive detection of bio molecules with the magneto-optic surface-plasmon-resonance sensor. Opt Lett. 2006;31(8):1085-1087.

22. Hermann C, Kosobukin V, Lampel G et al. Surface-enhanced magneto-optics in metallic multilayer films. Physical Review B. $2001 ; 64(23): 235422$.

23. Vila EF, Bendana SXM, González-Díaz JB et al. Surface plasmon resonance effects in the magneto-optical activity of $\mathrm{Ag}-\mathrm{Co}-\mathrm{Ag}$ trilayers. IEEE Transactions on Magnetics. 2008;44(11):3303-3306.

24. Robertson W, Fullerton E Re examination of the surface-plasma-wave technique for determining the dielectric constant and thickness of metal films. OSA B. 1989; 6(8):1584-1589.

25. Hickernell R, Dror S Long-range surface magnetoplasmons in thin nickel films. Opt Lett. 1987;12(8):570-572.

26. Newman D, Wears M, Matelon R, Hooper I Magneto-optic behaviour in the presence of surface plasmons. Journal of Physics: Condensed Matter. 2008;20(34):345230.

27. Breault-Turcot J, Masson J Nanostructured substrates for portable and miniature SPR biosensors. Anal Bioanal Chem. 2012;403(6):1477-1484.

28. Lambert $\mathrm{CH}$, Mangin S, Varaprasad BS et al. All-optical control of ferromagnetic thin films and nanostructures. Science. 2014;345(6202):1337-1340.

29. Rizal C, Moa B, Niraula B Ferromagnetic Multilayers: Magnetoresistance, Magnetic Anisotropy and Beyond (Review). J Magnetochemistry - an open access MDPI publication. 2016;2:30.

30. Rizal C, Niraula BB Ferromagnetic alloys: Magnetoresistance, microstructure, magnetism and beyond (review). J Nano-Electron Phys. 2015;7(4):04068.

31. Rizal C, Moa B, Brolo AG "Recent progress in ferromagnetic multilayerbased magnetoplasmonic devices for potential biomedical applications". The Canadian Medical and Biological Society Series pp. 2014;1-4.

32. Lu D, Kan JJ, Fullerton EE, Liu Z Enhancing spontaneous emission rates of molecules using nanopatterned multilayer hyperbolic metamaterials. Nat Nanotechnol. 2014;9(1):48-53.

33. Schubert M, Tiwald TE, Woollam JA Explicit solutions for the optical properties of arbitrary magneto-optic materials in generalized ellipsometry. Appl Opt. 1999;38(1):177-187.

34. Ertorer E, Vasefi F, Keshwah J et al. Large area periodic, systematically changing, multishape nanostructures by laser interference lithography and cell response to these topographies. J Biomed Opt. 2013;18(3):035002.

35. Menezes JW, Ferreira J, Santos MJ et al. Large-area fabrication of periodic arrays of nanoholes in metal films and their application in biosensing and Plasmonic-Enhanced photovoltaics. Advanced Functional Materials. 2010;20(22):3918-3924.

36. Fan M, Brolo AG Silver nanoparticles self assembly as SERS substrates with near single molecule detection limit. Physical Chemistry Chemical Physics. 2009;11(34):7381-7389. 
37. Maccaferri N, Gregorczyk KE, De Oliveira TV et al. Ultrasensitive and label-free molecular-level detection enabled by light phase control in magnetoplasmonic nanoantennas. Nat Commun. 2015;6:6150.

38. Gordon R, Brolo AG, Sinton D et al. Resonant optical transmission through hole-arrays in metal films: physics and applications. Laser \& Photonics Reviews. 2010;4(2):311-335.

39. Chin JY, Steinle T, Wehlus T et al. Nonreciprocal plasmonics enables giant enhancement of thin-film faraday rotation. Nat Commun. 2013;4:1599.

40. Temnov VV Ultrafast acousto-magneto-plasmonics. Nature Photonics 2012;6(11):728-736.

41. Hall DA, Gaster RS, Makinwa K et al. A 256 pixel magnetoresistive biosensor microarray in $0.18 \mu \mathrm{m}$ CMOS. IEEE J Solid-State Circuits. 2013;48(5):1290-1301.

42. Koets M, Van der Wijk T, Van Eemeren J Prins M Rapid DNA multianalyte immunoassay on a magneto-resistance biosensor. Biosensors Bioelectronics. 2009;24(7):1893-1898.

43. Wang SX, Li G Advances in giant magnetoresistance biosensors with magnetic nanoparticle tags: review and outlook. IEEE Transactions on magnetics. 2008;44(7):1687-1702.

44. Xu L, Yu H, Akhras MS, Han S, Osterfeld S, et al. Giant magnetoresistive biochip for DNA detection and HPV genotyping. Biosens Bioelectron. 2008;24(1):99-103.

45. De Boer B, Kahlman JA, Jansen TP et al. An integrated and sensitive detection platform for magneto-resistive biosensors. Biosens Bioelectron. 2007;22(9-10):2366-2370.

46. Han S, Yu H, Murmann B, Pourmand N, Wang SX A high-density magnetoresistive biosensor array with drift-compensation mechanism. Presented at 2007 IEEE International Solid-State Circuits Conference. Digest of Technical Papers. 2007
47. Graham DL, Ferreira HA, Freitas PP Magnetoresistive-based biosensors and biochips. Trends Biotechnol. 2004;22(9):455-462.

48. Schotter J, Kamp P, Becker A et al. Comparison of a prototype magnetoresistive biosensor to standard fluorescent DNA detection. Biosens Bioelectron. 2004;19(10):1149-1156.

49. Edelstein RL, Tamanaha CR, Sheehan PE, Miller MM, Baselt DR, et al. The BARC biosensor applied to the detection of biological warfare agents. Biosens Bioelectron. 2000;14(10):805-813.

50. Baselt DR, Lee GU, Natesan M, Metzger SW, Sheehan PE, et al. A biosensor based on magnetoresistance technology. Biosens Bioelectron. 1998;13(7-8):731-739.

51. Srinivasas B, Li Y, Jing Y et al. ("A detection system based on giant magnetoresistive sensors and high-moment magnetic nanoparticles demonstrates zeptomole sensitivity: potential for personalized medicine". Angewandte Chemie. 2009;48(15):2764-2767.

52. Jing Y, He S, Kline T, Xu Y, Wang JP High-magnetic-moment nanoparticles for biomedicine. Conf Proc IEEE Eng Med Biol Soc. 2009:4483-4486.

53. Petković I, Williams F, Bennaceur K et al. Edge magnetoplasmons in graphene. J Phys D. 2014;47(9):094010.

54. Liz Marzán LM, Murphy CJ, Wang J Nanoplasmonics. Chem Soc Rev. 2014;43(11):3820-3822.

55. Li Y, Zhang Q, Nurmikko AV, Sun S Enhanced magnetooptical response in dumbbell-like $\mathrm{Ag}-\mathrm{CoFe} 2 \mathrm{O} 4$ nanoparticle pairs. Nano Lett. 2005;5(9):1689-1692. 